\title{
Checkerboard-specific color aftereffects: A failure to find effects of perceptual organization
}

\author{
ALLEN E. MILEWSKI, JAMES IACCINO, and DAVID SMITH \\ DePaul University, Chicago, Illinois 60614
}

\begin{abstract}
Two experiments investigated the effects of differing perceptual organizations of reversible figures on McCollough aftereffects. Experiment 1 used colored checkerboard inducing stimuli and achromatic grating test stimuli. While some subjects tended to organize the checkerboards into rows and/or columns and others to organize them into obliques, these variations did not result in differences in aftereffect direction or magnitude. Experiment 2 induced an aftereffect with colored gratings and tested with checkerboards, gratings, and a reversible concentric octagon pattern. Perceptual organization had no effect on results for checkerboards, but was related to aftereffect strength for the octagon pattern. Indirect evidence suggests that, in the latter case, differences in aftereffect strength may have influenced the perceived organization, rather than vice versa. Finally, regardless of the specific organization perceived, spontaneous viewing of all test stimuli produced stronger aftereffects than were found when subjects reorganized the pattern. This may have resulted from a viewing strategy associated with reorganization, since similarly small aftereffects were found when subjects concentrated their attention on a single pattern element.
\end{abstract}

The McCollough effect can be elicited by exposing subjects to chromatic grating patterns. The color aftereffects are contingent upon the orientation of achromatic test gratings and are roughly complementary to the adapting color (McCollough, 1965; Skowbo, Timney, Gentry, \& Morant, 1975; Stromeyer, 1978). Most attempts to account for these aftereffects have proposed the adaptation of neural analyzers that are specific to both color and orientation (McCollough, 1965) and, in some cases, to other features, such as movement direction (Hepler, 1968), curvature (Riggs, 1973), and spatial frequency (Stromeyer, 1972). These explanations are based on relatively low-level, involuntary mechanisms. Such interpretations are enticing, because, in most cases, the effects are associated with retinal stimulus properties rather than the properties as perceived by the subject (Harris, 1970; Stromeyer, 1978).

In contrast, however, recent reports have presented evidence interpreted as suggesting cognitive control over the McCollough effect. Specifically, Jenkins and Ross (1977) and Uhlarik, Pringle, and Brigell (1977) have used reversible figures as test patterns. Such patterns alternate between two distinct perceptual organizations. Alternation occurs spontaneously and/or voluntarily despite an invariant stimulus. Following inspection with colored gratings, theses studies have found that the presence of aftereffect hues is related to the particular perceptual organization that the subject experiences. For example, Jenkins and Ross (1977) found strong color aftereffects when subjects organized their concentric-square test pattern into four triangular sections of stripes. But little or no aftereffect was observed when the test pattern was organized as concentric squares. These results have important implications for our understanding of McCollough aftereffects. They suggest that the effects may not be as inflexible or as dependent on the physical stimulus properties as neural analyzer explanations would predict. Instead, they argue that higher order cognitive factors must be included for a complete explanation.

However, conclusions from the results of previous studies with reversible figures must be tentative for several reasons. The first has to do with the distinction between the empirical results obtained and the internal condition of the subjects that is inferred from them. Both Jenkins and Ross (1977) and Uhlarik et al. (1977) used reversible figures only as test stimuli and not as inducing stimuli. It would seem likely that factors influencing the test phases of an aftereffect experiment may have a more tenuous link to the causality of the aftereffect than would those influencing its induction. Hence, this study explores the consequences of reversible figure organization both during induction and test. The second reason for caution concerns the fact that both previous studies used somewhat similar reversible figures. The generality of perceptual organization effects to other stimuli is not known from the existing literature.

The present study was designed to investigate the role of perceptual organization for McCollough aftereffects with checkerboard stimuli. Checkerboard patterns may be considered reversible figures. An observer may perceptually organize a checkerboard as a series of rows, columns, or obliques, but 
it is not possible to see more than one organization at a time. Several studies have shown reliable McCollough aftereffects using checkerboard inducing or test stimuli (Green, Corwin, \& Zemon, 1976; May, Agamy, \& Matteson, 1978; May \& Matteson, 1976). Except at very low spatial frequencies, these studies have found that the orientation in a checkerboard that is most relevant for McCollough aftereffects is not that of the element edges, but rather that of the fundamental Fourier components (which are at 45 and 135 deg to the element edges; Kelly, 1976). For example, following inspection of green oblique and red vertical gratings, an upright checkerboard appeared pinkish, while a rotated checkerboard (comprised of diamond-shaped elements) appeared greenish (May \& Matteson, 1976).

Checkerboard stimuli provide an especially strong test of the role of perceptual organization in McCollough aftereffects. This is because, under appropriate conditions, the aftereffect hue itself should reverse, depending on whether the pattern is organized as verticals/horizontals or as obliques. In contrast, the previous studies of perceptual organization were designed so that changes in organization should produce changes only in the magnitude of the aftereffect, a measure which may depend on many other factors. None of the previous studies using checkerboards controlled or measured the perceptual organizations experienced by subjects.

\section{EXPERIMENT 1}

Experiment 1 was designed to investigate the effects of perceptual organization of induction stimuli on McCollough aftereffects. Perceptual organizations were recorded while subjects viewed colored checkerboard inducing stimuli. Aftereffects for subsequent achromatic test gratings were then examined for correlations with the prior perceptual organizations.

\footnotetext{
Method

Subjects. Twenty-six subjects participated in Experiment 1. Fifteen were undergraduates taking a course in experimental psychology and 11 were graduate students in psychology. All subjects were naive with regard to McCollough aftereffects, and all reported normal or corrected-to-normal vision.

Stimuli. Stimuli were Ortholith high-contrast photographic slides projected onto a white screen and viewed from a distance of approximately $285 \mathrm{~cm}$. All stimulus patterns were shown within a circular area, $40 \mathrm{~cm}$ in diameter. Induction stimuli were regular and diamond-element checkerboards colored by Wratten gelatin filters (No. 26 magenta and No. 55 green). Luminances were 45.5 and $78.2 \mathrm{~cd} / \mathrm{m}^{2}$, respectively, with contrasts of approximately .80 and .85 . During the test, the circular stimulus area was divided into quadrants, each containing an achromatic square-wave grating of different orientation: vertical, horizontal, and the two obliques. Oblique lines were never in adjacent quadrants. These stimuli were similar to those used by Green et al. (1976). Seven different test stimuli were used with spatial frequencies of 1.8, 2.1, 2.6,
}

3.1, 3.6, 5.0, and 8.4 cycles/deg. Compared with the checkerboard element size, these stimuli represent $-1,-.8,-.5,-.2,0, .5$, and 1.2 octave changes. The luminance of these achromatic test stimuli was $342 \mathrm{~cd} / \mathrm{m}^{2}$, and the contrast was .94 .

Procedure. The subjects were initially presented with the seven achromatic test stimuli for a pretest rating. For each stimulus, the subjects were instructed to indicate, in a forced-choice manner, whether the quadrants appeared greenish or pinkish and also to rate the color strength on a 5-point scale. For this purpose, the subjects judged the oblique grating quadrants as a single unit and similarly rated the quadrants of vertical and horizontal gratings. Following the pretest, induction began by alternating between two induction stimuli. Each stimulus was presented for 6010 -sec periods. At the end of each period, the subjects indicated their dominant perceptual organizations. This was accomplished by their briefly looking away from the inducing stimulus and marking boxes labeled either "Diagonals" or "Rows and Columns" on a data sheet. Finally, tests of color aftereffects were conducted that were identical to the pretest phase.

The subjects were tested in groups of 2 to 14 in a darkened room. Half the subjects received red regular checkerboards and green diamond-element checkerboards during induction and a single random order of stimuli during the test. The other subjects received the opposite pairing of induction stimuli and a different random order of test stimuli.

\section{Results and Discussion}

McCollough aftereffects. For each test stimulus, the perceived color and rated strength of the oblique quadrants was compared with those of the verticalhorizontal quadrants. If both quadrant pairs were perceived as being the same color, their color strength ratings were subtracted. If pairs were perceived as different colors, their strength ratings were added. These differences were given a positive score if the results were in the direction typically found with checkerboards (e.g., obliques pinker than verticalhorizontals following induction with red regular checkerboards; May \& Matteson, 1976). A negative sign was given if the direction obtained was opposite to that typically found. Finally, aftereffect difference scores were computed for each subject separately for each stimulus by subtracting the score for each test stimulus for the pretest score for that stimulus.

Figure 1 shows the mean aftereffect difference scores for each test-stimulus spatial frequency. Comparing scores at each spatial frequency with zero by means of $t$ tests showed a significant aftereffect at .5 octaves $[\mathrm{t}(25)=2.41, \mathrm{p}=.02]$ and 1.2 octaves relative to the periodicity of checkerboard elements $[\mathrm{t}(25)=2.02, \mathrm{p}=.05]$. There was also a marginal effect at 0 octaves $[\mathrm{t}(25)=1.9, \mathrm{p}=.06]$. No other differences approached significance. These results concur with those of May and Matteson (1976) in two ways. First, the predominance of positive scores shows that, on the average, McCollough aftereffects for checkerboards transfer according to the fundamental Fourier component rather than according to element edges. Second, although not statistically 
significant, these results suggest that the best transfer occurs when the test stimulus spatial frequency is .5 octaves above the checkerboard period.

Perceptual organization. For each subject, the number of times each inducing checkerboard was seen as diagonals or as rows and/or columns was tallied. The mean tallies and standard deviations are shown in Table 1. Generally, the subjects tended to organize the regular checkerboard as diagonals more often than as columns and rows, while the opposite was true for the diamond-element checkerboards. Despite this general tendency, the subjects showed great variation in their perceptual organizations. The standard deviations in Table 1 are large, and the tallies in each cell have a large range. This large range of perceived organizations provides for a reasonable scale on which to compare the McCollough aftereffects. Table 2 shows the Pearson product moment correlations between subjects' aftereffect difference score and both the number of periods the regular checkerboard was seen as rows and columns and the number of periods the diamond-element checkerboard was seen as rows and columns. As can be

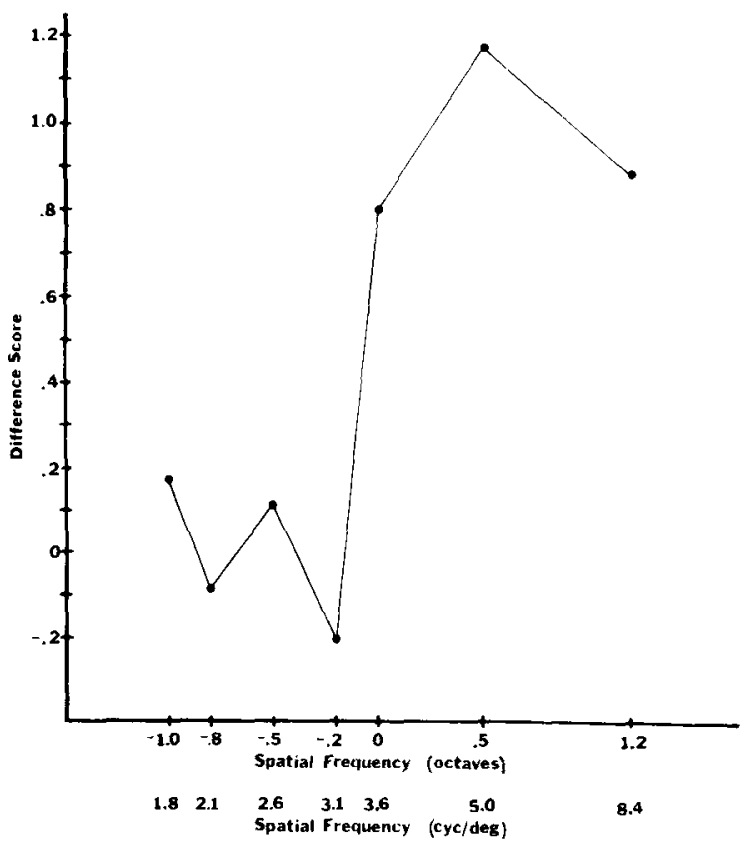

Figure 1. McCollough effect difference score as a function of test grating frequency for Experiment 1.
Table 2

Pearson's Correlations of Aftereffect Difference Scores with the Frequency of Organizing the Inducing Stimuli as Rows and/or Columns

\begin{tabular}{|c|c|c|c|c|}
\hline \multirow{2}{*}{$\begin{array}{c}\text { Test Stimuli } \\
\text { (Octaves) }\end{array}$} & \multicolumn{2}{|c|}{$\begin{array}{c}\text { Regular } \\
\text { Checkerboard }\end{array}$} & \multicolumn{2}{|c|}{$\begin{array}{l}\text { Diamond-Element } \\
\text { Checkerboard }\end{array}$} \\
\hline & I & $\mathrm{p}$ & I & $\mathrm{p}$ \\
\hline .0 & .07 & .36 & -.04 & .40 \\
\hline .5 & .20 & .16 & .01 & .45 \\
\hline 1.2 & .11 & .29 & .11 & .28 \\
\hline
\end{tabular}

seen for the 0-, .5-, and 1.2-octave test stimuli, there was no reliable relationship between perceived organization of the inducing checkerboards and the subsequent color-aftereffect magnitude.

It is possible that the failure to obtain significant relationships may be attributed partly to the insensitivity of the measures employed. However, the same measures did produce aftereffect results consistent with those of previous studies (Green et al., 1976; May \& Matteson, 1976). Moreover, even at a gross level of analysis, a relationship between aftereffects and organization failed to appear. Perceptual organization effects would predict negative difference scores for subjects organizing the regular checkerboard as rows and columns. Only six subjects had negative difference scores at $0, .5$, or 1.2 octaves, and only two of these organized the regular checkerboard as rows and/or columns more than 30 times (out of 60 periods). Finally, the present results are consistent with a recent report by White (1979), who found, with noncheckerboard stimuli, that aftereffects were predicted by the main Fourier components and not by the organization as perceived by the subjects.

\section{EXPERIMENT 2}

The conclusion of Experiment 1 for checkerboards is discrepant with previous studies which have used other reversible figures (Jenkins \& Ross, 1977; Uhlarick et al., 1977). In addition to the differing stimulus conditions employed, there are several possible explanations for the discrepancy. First, while the present study used a reversible figure as the induction stimulus followed by nonreversible test gratings, the previous studies had adapted with gratings and had

Table 1

Mean and Standard Deviation of Perceptual Organization Tallies $(N=26)$

\begin{tabular}{|c|c|c|c|c|c|c|}
\hline \multirow[b]{3}{*}{ Stimulus } & \multicolumn{6}{|c|}{ Perceived Organization } \\
\hline & \multicolumn{3}{|c|}{ Columns and/or Rows } & \multicolumn{3}{|c|}{ Diagonals } \\
\hline & Mean & SD & Range & Mean & SD & Range \\
\hline Regular Checkerboard & 26.50 & 19.37 & $1-60$ & 33.65 & 19.54 & $0-59$ \\
\hline Diamond-Element Checkerboard & 39.08 & 20.08 & $0-60$ & 19.42 & 19.95 & $0-60$ \\
\hline
\end{tabular}


tested for aftereffects with reversible figures. It is possible that while the formation of McCollough aftereffect may depend solely on the physical stimulus parameters, the appearance of the aftereffects, once formed, may depend on the perceived organization of the test stimulus. Experiment 2 investigated this possibility by using gratings as induction stimuli followed by reversible test stimuli. A second issue pertaining to the previous studies concerns the causal relationship between the color aftereffect magnitude and the way in which subjects spontaneously organize the reversible test pattern. Given the design and stimuli previously employed, it is difficult to determine whether the organization that was spontaneously perceived had a causal effect on the aftereffect magnitude, or whether individual differences in the McCollough aftereffect magnitude emphasized a specific organization for some subjects and not for others. Checkerboard test stimuli circumvent this problem, since the perceived color of a checkerboard should not affect its perceived organization. Experiment 2 attempted to investigate this issue by employing both checkerboard test patterns and a pattern of concentric octagons that was analogous to the concentric square pattern used by Jenkins and Ross (1977).

A final issue concerns the possibility that, regardless of the specific organization that is perceived, McCollough aftereffects may be stronger for reversible figures that are viewed in their spontaneous or dominant organization compared with those that are reorganized into a less dominant organization. For example, Jenkins and Ross (1977) found that, after induction, subjects tended to organize the pattern of concentric squares into triangular segments of alternating hues. When forced to switch into a concentricsquare organization, the McCollough aftereffect faded. It is possible that the perceived organization per se had little to do with the decrease in aftereffect magnitude. Instead, the process of switching to a nondominant organization itself may have caused the decrease. No clear conclusion has been reached regarding what subjects must do in order to switch the organization of reversible figures. But, introspectively, it appears that concentrating one's attention on some part of the pattern aids its reorganization. Experiment 2 investigated this final issue in two ways. First, McCollough aftereffects were measured both for subjects' spontaneous organizations and for the alternative organization (i.e., after being instructed to reorganize). Second, McCollough aftereffects were assessed after subjects had been instructed to concentrate their attention on some part of the test patterns. This latter procedure, it was hoped, would mimic part of the strategy involved in reorganizing reversible figures without stressing any specific organization.

\section{Method}

Subjects. The subjects were 34 undergraduate students enrolled in an introductory psychology class. Data from an additional 8 subjects were not analyzed because they failed an initial screening for presence of McCollough aftereffects on gratings (see Procedure).

Stimuli. The inducing stimuli were four rear-projected Ortholith high-contrast photographic slides of square-wave gratings (4.4 cycles/deg). Vertical and horizontal gratings were projected through a Wratten No. 26 (magenta) filter, and the two oblique gratings were projected through a Wratten No. 55 (green) filter. The luminances were 54.2 and $85.5 \mathrm{~cd} / \mathrm{m}^{2}$, respectively. Each stimulus was quadrant-shaped, $8.5 \mathrm{~cm}$ on a side, and viewed from approximately $300 \mathrm{~cm}$. The contrasts were approximately .81 and .88. Each stimulus was presented in a darkened room for $15-\mathrm{sec}$ periods. Total adaptation time was $20 \mathrm{~min}$. Achromatic test stimuli were printed on sheets of white paper that were held at a normal reading distance and aligned with the body axis by the subjects. The test stimuli are shown in Figure 2. The checkerboard and grating stimuli were $25-\mathrm{mm}$ squares. The concentric octagon pattern measured $34 \mathrm{~mm}$ laterally. At a typical reading distance, the spatial frequency of the printed test stimuli was roughly the same as that for the projected inducing stimuli. The illuminance at the printed test materials was approximately $269 \mathrm{~lx}$.

Procedure. Prior to induction of McCollough aftereffects, the subjects were presented with the achromatic checkerboard and concentric octagon patterns printed in an instruction booklet. They were instructed that the patterns could be seen as reversible figures. The checkerboards could be organized into either a series of vertical and/or horizontal stripes or a series of oblique stripes. The octagon pattern could be organized into either concentric octagons or into eight triangular segments, each pointing toward the center. The subjects indicated their spontaneous or dominant organization for the octagon and for the regular and diamondelement checkerboard sections separately. They were then instructed to attempt to reorganize each pattern to the alternative organization and to indicate the difficulty they had in doing so
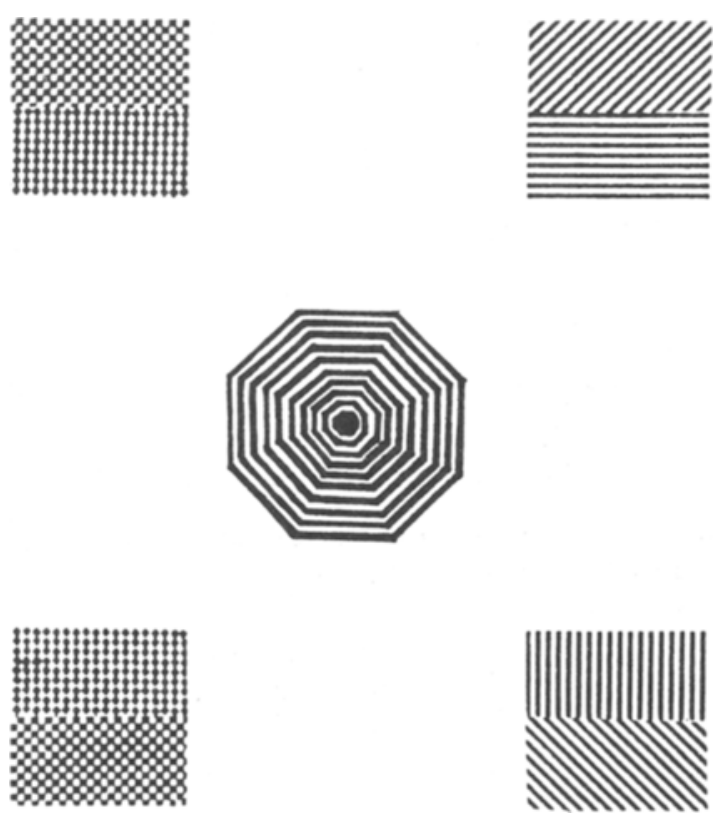

Figure 2. Test stimuli employed in Experiment 2. 
on a 4-point scale. Following this rating, the 20 -min induction segment began.

The subsequent testing period consisted of four parts. The first part included initial aftereffect screening and instructions on the aftereffect scale to be used. The subjects were presented with the printed grating stimuli (vertical and oblique) and asked to note if any colors appeared. Those who responded positively were asked to indicate which part appeared greenish and which pinkish. All of these subjects responded in a way consistent with the typical McCollough aftereffect, and only these subjects continued in the experiment. The subjects were introduced to an 11-point scale to be used to rate color aftereffects. Negative 1 to negative 5 indicated increasing greenish appearance, while positive 1 to positive 5 indicated increasing pinkishness. A rating of 0 indicated no color. In an attempt to have the entire scale utilized, the subjects were instructed that the magnitude of color experienced in the initial vertical and oblique gratings patterns were to be called negative 3 and positive 3 , respectively. These gratings were then used as an individually defined standard and referred to during subsequent ratings.

The second part of the testing period involved the checkerboard patterns. Initial McCollough aftereffect ratings were obtained from the regular and diamond-element sections separately, along with an indication of the spontaneous or dominant perceptual organization. The subjects were then asked to reorganize the patterns, indicate the difficulty in doing so on a 4-point scale, and also to rate the color aftereffects under the new organization.

The third part was identical to the second part, with the exception that the octagon patterns were used. Subjects rated McCollough aftereffects in each of the eight sections of the pattern separately, regardless of their perceptual organization.

The fourth part of the testing period involved re-presentation of the checkerboards, octagons, and gratings. The subjects were instructed once again to rate the McCollough aftereffects. But, rather than having them organize the pattern one way or another, they were instructed to concentrate their attention closely on a single checkerboard element or stripe (for the octagon and grating). Half the subjects received this part at the end of the testing period, while the other half received it immediately following the first part. The instructions for this attentional manipulation are given in the Appendix.

\section{Results and Discussion}

In order to assess aftereffect magnitude, difference scores were computed. For the octagon and grating patterns, the mean color strength ratings for the vertical-horizontal components were subtracted from that of the oblique components. For the checkerboards, the rating for the diamond-element checkerboard was subtracted from that of the regular checkerboard. Hence, a positive difference score indicates results consistent with the typical McCollough aftereffect findings.

Checkerboard patterns. Organizational and ease of reorganization data were obtained both prior to and following induction. Prior to induction, 24 subjects organized the regular checkerboard as verticalhorizontals, while 20 subjects organized it as obliques. Again, during postinduction spontaneous viewing, 24 subjects organized the regular checkerboard as vertical-horizontals and 10 subjects organized it as obliques. Twelve subjects switched their spontaneous organization from pre- to postinduction viewing, six from obliques to vertical-horizontals, and six from vertical-horizontals to obliques. Similar results were obtained for the diamond-element checkerboard. Nineteen subjects viewed it as vertical-horizontals and 15 viewed it as obliques, with no differences between pre- and postinduction periods. Four subjects switched from vertical-horizontals to obliques and four switched in the opposite direction. The rating of ease of reorganization showed no difference between pre- and postinduction periods or between stimuli. Also, it did not depend on whether subjects spontaneously organized the stimuli as vertical/horizontals or as obliques.

Aftereffect color strength ratings were analyzed for differences between specific organizations (vertical/ horizontal vs. obliques), and also for differences between viewing modes (spontaneous viewing vs. reorganized viewing). The data were first collapsed across the spontaneous and reorganized viewings, and a within-subjects $t$ test was performed. Thus, aftereffect magnitude for checkerboard test stimuli that were organized as vertical/horizontals was compared with those organized as obliques. No reliable differences in aftereffect magnitude were found between the two organizations $[\mathrm{t}(33)=.57, \mathrm{p}=.6]$. A withinsubjects $t$ test was employed to assess viewing mode differences after the data were collapsed across vertical/ horizontal vs. oblique organizations. There was a significantly stronger aftereffect under spontaneous viewing compared with reorganized viewing [t(33) $=$ $2.09, p=.04]$. This effect is shown in the first panel of Figure 3. Furthermore, there was no evidence for interactions between viewing mode and the specific organization. The design of the experiment did not permit a clear general test of interactions. Moreover, since the perceived organization of regular and diamond-element checkerboards were not mutually exclusive, interactions had to be assessed twice: once according to the perceived organization of the regular checkerboard and once according to the organization of the diamond-element checkerboard. Nonetheless, between-subjects $t$ tests indicated that aftereffect magnitude under spontaneous viewing did not depend on whether the regular checkerboard was organized as vertical/horizontals or as obliques [t(33) $=1.61, p=.10]$, or on how the diamond-element checkerboard was organized $[\mathrm{t}(32)=.94, \mathrm{p}=.60]$. Similarly, aftereffect magnitude under reorganized viewing did not depend on how the regular checkerboard was organized $[t(32)=1.41, p=.16]$ or on the specific organization of the diamond-element checkerboard $[\mathrm{t}(32)=1.14, \mathrm{p}=.25]$.

In summary, the results for checkerboards are consistent with those of Experiment 1 in showing no relationship between McCollough aftereffect direction or magnitude and the way in which the stimuli are perceptually organized. Moreover, the fact that mean organizational data for spontaneous viewing 
was identical for pre- and postinduction periods suggests that the induction of a McCollough aftereffect does not alter the perceived organization of checkerboards in any regular way. Finally, the significant effect of viewing mode on aftereffect magnitude suggests that the act of perceptually reorganizing checkerboards per se does produce a decrement in aftereffect magnitude.

Octagon pattern. Organizational data for the octagon pattern prior to induction indicated that 19 subjects spontaneously organized the pattern as concentric octagons, while 15 subjects organized it as triangular sectors. Following induction, however, nine subjects spontaneously organized it as concentric octagons, while 25 subjects organized it as triangular sectors. While 3 subjects switched from triangular to concentric octagon organizations between pre- and postinduction periods, 13 subjects switched from concentric octagons to a triangular-sector organization. The change in organization between pre- and postinduction periods leading to a predominance of the triangular sector organization after induction was significant by McNemar's test for correlated proportions $(p=.005)$. Ease of reorganization ratings during pretest did not differ according to the specific spontaneous organization. However, following induction, subjects who had spontaneously organized the pattern as triangular sectors found it significantly more difficult to reorganize their percepts than did subjects who had spontaneously organized the pattern as concentric octagons $[\mathrm{t}(32)=3.45, \mathrm{p}=.002]$.

The aftereffect color strength ratings for the octagon pattern were analyzed both for differences between specific organizations and foi differences between viewing modes. As for the checkerboards, spontaneous viewing of the octagon test stimulus resulted in significantly stronger aftereffects than did reorganized viewing $[\mathrm{t}(33)=3.50, \mathrm{p}=.002]$. This difference is shown in the middle panel of Figure 3. However, in contrast with the checkerboard data, the

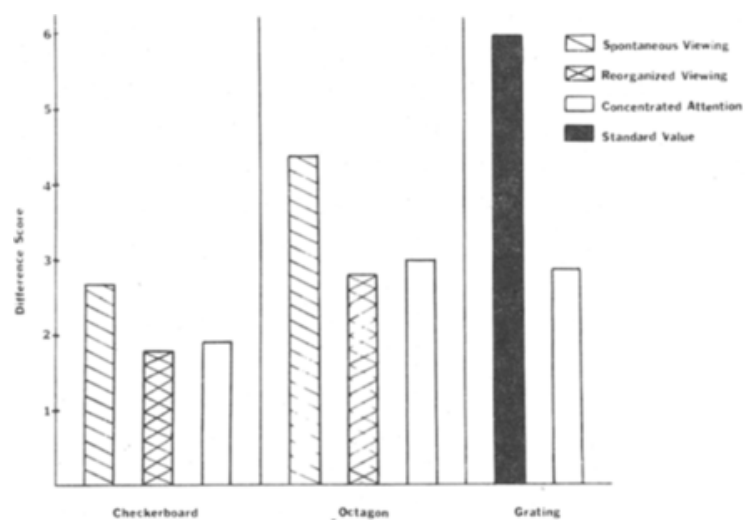

Figure 3. McCollough effect difference scores as a function of test stimulus and viewing mode for Experiment 2.

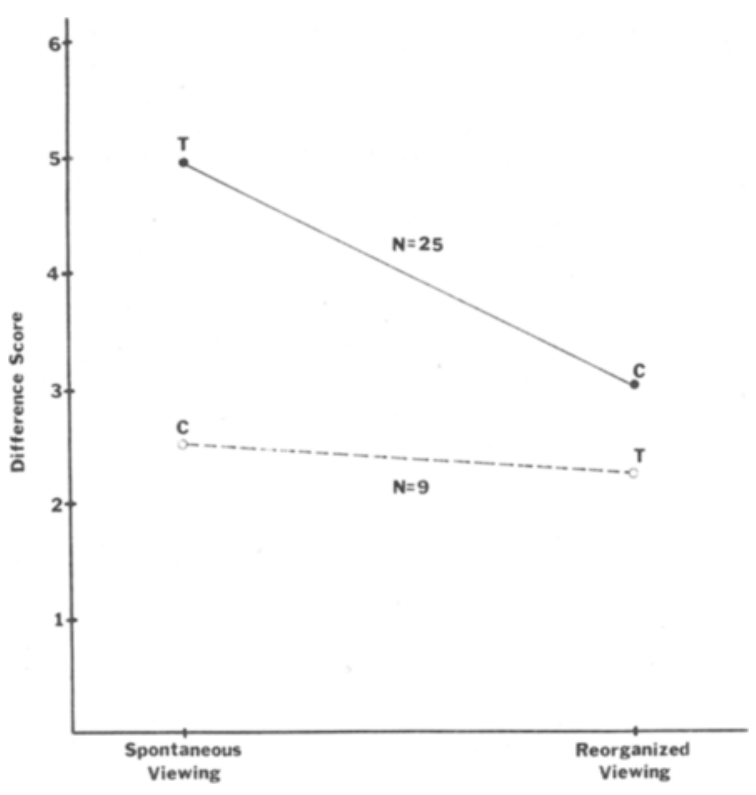

Figure 4. McCollough effect difference scores for the octagon test stimulus for Experiment 2. $C$ indicates scores under the concentric octagon organization; $T$ indicates scores under the triangular sector organization.

octagon stimulus also resulted in a significant effect of stimulus organization [t(33) $=3.24, \mathrm{p}=.003]$. Specifically, when the data were collapsed across viewing mode, a stronger aftereffect was obtained when the subjects organized the stimulus as triangular sectors rather than as a concentric octagon. This finding with the concentric octagon pattern is consistent with that of Jenkins and Ross (1977), who used a concentric square pattern. Their implicit conclusion, however, that perceptual organization has a causal effect on McCollough aftereffect magnitude, can be questioned. Instead, while the present study provides no direct evidence, there is some indirect evidence suggesting that individual differences in aftereffect magnitude may have caused the differences in perceived organization. First, the organizational and ease of reorganization data described above suggest that the induction of a McCollough aftereffect affects the perceptual organization of the octagon test stimulus. It does so by making the triangular-sector organization more common and less easily reorganized than it is during preinduction viewing. Second, unlike the case of the checkerboards, the octagon pattern data suggest some interaction between viewing mode and specific organization. Specifically, during spontaneous viewing, the triangular-sector organization resulted in stronger aftereffects than did the concentric octagon or organization $[\mathrm{t}(32)=3.09, \mathrm{p}=.006]$, but there was no difference due to organization during reorganized viewing $[t(32)=.80, p=.54]$. This interaction is shown in Figure 4. It is possible to interpret this finding by assuming that those nine subjects who 
spontaneously organized the postinduction pattern as concentric octagons did so partly because their aftereffect strength for the complex patterns was small compared with that for stripes. Some evidence for this interpretation comes from an analysis comparing aftereffect magnitude for the checkerboard stimuli of the nine subjects who organized the octagon as concentric octagons with that of the 25 subjects who organized it as triangular sectors. While only marginally significant, this analysis suggests that the subjects who organized the octagon as triangular sectors showed somewhat stronger aftereffects on the checkerboard patterns $[F(1,32)=3.29, p=.07]$.

In summary, the data suggest that the octagon stimulus and checkerboard stimuli are similar in that spontaneous viewing results in stronger McCollough aftereffects than does reorganized viewing. Unlike the checkerboards, however, the octagon pattern showed differences in aftereffect magnitude as a function of organization. While this finding is consistent with previous studies on reversible figures, there is some suggestion that the effect was artifactual and due to individual differences in aftereffect strength, regardless of organization.

Effect of concentrated attention. Aftereffect magnitude was also measured while subjects attempted to concentrate their attention on a single element of the checkerboard, octagon, and stripes. The results of this procedure are relevant in two ways. First, since half the subjects received this condition at the beginning of the test period and half received it at the end, the differences between these groups can be used to assess the decay of aftereffects across the testing period. There were no significant differences between these groups for any of the stimuli employed. While the failure to find evidence of decay may be a function of the individually defined standard employed, it does argue against the possibility that the spontaneous vs. the reorganized viewing effect discussed above is due merely to decay across time. Second, the original intent of this condition was to attempt to mimic part of the strategy involved in pattern reorganization. The results for each pattern are shown in Figure 3. For each pattern, aftereffect magnitude under concentrated attention was nominally lower than for spontaneous viewing. The difference was significant for octagons $[\mathrm{t}(33)=4.65, \mathrm{p}<.001]$ and for stripes $[\mathrm{t}(33)=5.186, \mathrm{p}<.001$, compared with the standard value of 6], but failed to reach significance for checkerboards $[\mathrm{t}(33)=1.27, \mathrm{p}=.2]$.

\section{GENERAL DISCUSSION}

The clearest and most important finding of the present investigation was the failure to find effects of perceptual organization on McCollough aftereffects with checkerboards. Checkerboards provide a unique test of perceptual organization effects, since differences in perceived organization should have resulted in changes in the aftereffect hue itself rather than simply in the magnitude of the aftereffect. The present investigation found no evidence for such a change, regardless of whether checkerboards were used as inducing or as test stimuli. Rather, the present findings replicate those of previous McCollough aftereffect studies with checkerboards (Green et al., 1976; May \& Matteson, 1976). These studies indicate that, on the average, aftereffects transfer to the primary Fourier component of checkerboards. Moreover, for checkerboards, the present investigation found no relationship between individual differences in perceived organization and aftereffect direction or strength.

In contrast with the checkerboard findings, Experiment 2 found that a test pattern of concentric octagons resulted in stronger McCollough aftereffects when organized into triangular sectors then when organized into a concentric octagon. This finding replicates those of studies using similar reversible figures (Jenkins \& Ross, 1977; Uhlarik et al., 1977). There may be several differences between checkerboards and the concentric octagon pattern that could explain the differences in results. One difference of special interest concerns the issue of causal direction. For the octagon, the presence of aftereffect color could influence the perceived organization by accentuation of the triangular sectors. A similar influence is difficult to imagine for the checkerboard. From aftereffect results alone, it is difficult to determine the direction of causality. Previous studies have concluded that the perceived organization affects aftereffect magnitude. Both organizational data and indirect aftereffect findings in the present investigation suggest the opposite for the octagon pattern: that the aftereffect strength may have influenced the organization.

The second important finding of the present investigation is that spontaneous viewing of the test pattern resulted in stronger McCollough aftereffects than were found while subjects perceptually reorganized the pattern. This was true regardless of the specific organization perceived. Results in the same direction were found when subjects were asked to concentrate their attention on a single pattern element. This decrement in aftereffect strength appears to be related to a particular viewing strategy that may be associated with perceptual organization to a nondominant organization. To our knowledge, no previous investigation has reported a similar phenomenon. Skowbo et al. (1974) and Stromeyer (1972) have shown that prolonged scanning of the test stimulus can cause a decrease in aftereffect magnitude. However, in the present study, subjects never viewed the test patterns for more than a few minutes. It is doubtful that these findings are directly related. It is not clear 
what type of mechanism could account for the aftereffect decrement with concentrated attention, but viewing strategies deserve attention in future research on McCollough aftereffects.

The present investigation has separated two factors that are associated with McCollough aftereffects for reversible figures. First, the aftereffect strength itself can influence perceptual organization by accentuating one organization over another. Second, the act of reorganizing a reversible figure can produce a decrement in aftereffect strength, regardless of the specific organization experienced. Neither of these factors could be considered true perceptual organization effects. However, it is possible that one or both of these factors may have influenced the results of previous studies. To what extent the influence of these factors has confounded their conclusions is not clear. Recently, Finke and Schmidt $(1977,1978)$ have shown perceptual organization effects that cannot be accounted for by these factors alone. These investigators found, in a highly selective group of subjects, that merely imagining a perceptual organization can influence aftereffects results even when there is no pattern stimulus present. What is clear from the present checkerboard data is that the influences of perceptual organization are not easily found for all reversible figures.

\section{REFERENCES}

Finke, R. A., \& Schmidt, M. J. Orientation-specific color aftereffects following imagination. Journal of Experimental Psychology: Human Perception and Performance, 1977, 3, 599-606.

Finke, R. A., \& SchmidT, M. J. The quantitative measure of pattern representation in images using orientation-specific color aftereffects. Perception \& Psychophysics, 1978, 23, 515-520.

Green, M., Corwin, T., \& Zemon, V. A comparison of Fourier analysis and feature analysis in pattern-specific color aftereffects. Science, 1976, 192, 147-148.

Harris, C. S. Effect of viewing distance on a color aftereffect specific to spatial frequency. Psychonomic Science, 1970, 21, 350.

Hepler, N. K. A motion-contingent aftereffect. Science, 1978, $162,376-377$.
Jenkins, B., \& Ross, J. McCollough effect depends upon perceived organization. Perception, 1977, 6, 399-400.

Kelly, D. H. Pattern detection and the two-dimensional Fourier transformation: Flickering checkerboards and chromatic mechanisms. Vision Research, 1976, 16, 277-289.

May, J. G., Agamy, G., \& Matteson, H. H. The range of spatial frequency contingent color aftereffects. Vision Research, 1978, 18, 917-921.

MAY, J. G., \& Matteson, H. H. Spatial frequency-contingent color aftereffects. Science, 1976, 192, 145-147.

McCollough, C. Color adaptation of edge-detectors in the human visual system. Science, 1965, 149, 1115-1116.

Riggs, L. A. Curvature as a feature of pattern vision. Science, 1973, 181, 1070-1072.

Skowbo, D., Timney, B. N., Gentry, T. A., \& Morant, R. B. McCollough effects: Experimental findings and theoretical accounts. Psychological Bulletin, 1975, 82, 497-510.

Stromeyer, C. F., III. Edge-contingent color aftereffects: Spatial frequency specificity. Vision Research, 1972, 12, 717-733.

Stromeyer, C. F., III. Form-color aftereffects in human vision. In R. Held, H. W. Leibowitz, \& H. L. Teuber (Eds.), Handbook of sensory physiology (Vol. 8) Perception. Berlin: SpringerVerlag, 1978.

Uhlarik, J., Pringle, R., \& Brigell, M. Color aftereffects contingent on perceptual organization. Perception \& Psychophysics, 1977, 22, 506-510.

White, K. D. Contingent after effects, "plaid patterns," and spatial filtering. Journal of the Optical Society of America, 1979, $69,1419$.

\section{APPENDIX}

The instructions for Part 4 of Experiment 2 were: "Rate the color of each of the components of the following figures. This time I want you to view the components differently. I want you to concentrate very hard. Do not merely gaze at the figures. Focus your attention on the individual checks (for the checkerboards) and the lines (in the other patterns). Concentrate! When you are really concentrating, you may notice that the colors will either remain the same, darken, wash out, or reverse (i.e., go from red to green or from green to red). When you have your attention really focused, rate the color of the components in comparison of those of the torn-out page. Take your time."

(Received for publication January 2,1980; revision accepted May S, 1980.) 